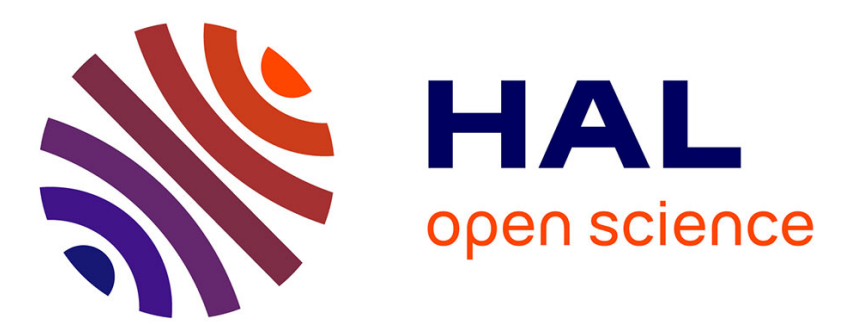

\title{
L'effet Peltier appliqué à la conception et la réalisation d'un nouveau capteur de débit massique
}

\author{
F. Wattiau, Etienne Gaviot, P. Thery
}

\section{To cite this version:}

F. Wattiau, Etienne Gaviot, P. Thery. L'effet Peltier appliqué à la conception et la réalisation d'un nouveau capteur de débit massique. Journal de Physique III, 1993, 3 (8), pp.1697-1709. 10.1051/jp3:1993230 . jpa-00249031

\section{HAL Id: jpa-00249031 https://hal.science/jpa-00249031}

Submitted on 1 Jan 1993

HAL is a multi-disciplinary open access archive for the deposit and dissemination of scientific research documents, whether they are published or not. The documents may come from teaching and research institutions in France or abroad, or from public or private research centers.
L'archive ouverte pluridisciplinaire HAL, est destinée au dépôt et à la diffusion de documents scientifiques de niveau recherche, publiés ou non, émanant des établissements d'enseignement et de recherche français ou étrangers, des laboratoires publics ou privés. 
Classification

Physics Abstracts

$06.70 \mathrm{M}-47.60$

\title{
L'effet Peltier appliqué à la conception et la réalisation d'un nouveau capteur de débit massique
}

\author{
F. Wattiau, E. Gaviot et P. Thery \\ Laboratoire de Mesures Automatiques, USTLFA, Bât. P3, 59655 Villeneuve d'Ascq Cedex, \\ France
}

(Reçu le 17 avril 1992, révisé le 25 mars 1993, accepté le 30 avril 1993)

\begin{abstract}
Résumé. - Le travail présenté est relatif à la conception et la réalisation d'un nouveau capteur de débit massique basé sur l'effet Peltier. Le capteur en forme de circuit imprimé bimétallique. comporte plusieurs pistes métalliques continues parallèles entre elles et partiellement recouvertes par un grand nombre de dépôts électrolytiques de nature différente. Un tel circuit se comporte comme un circuit thermoélectrique classique dans lequel les parties homogènes non recouvertes par le dépôt électrolytique constituent le premier conducteur du couple, les parties plaquées constituant le second conducteur. La méthode de mesure consiste à faire passer un courant électrique dans une ou plusieurs pistes constituant le circuit émetteur de façon à générer par effet Peltier des gradients thermiques qui seront détectés par un circuit détecteur identique placé à proximité. Dans le cas particulier d'un fluide au repos, la f.e.m. détectée par le circuit détecteur dépend de la conductivité thermique du fluide. Lorsque le fluide est en mouvement relatif par rapport au circuit, il est possible de détecter une f.e.m. dépendant en grandeur et en signe de la vitesse d'écoulement du fluide. A titre d'application un capteur de débit massique a été réalisé en plaçant les circuits thermoélectriques dans une canalisation parcourue par le fluide en mouvement.
\end{abstract}

Abstract. - The present paper deals with design and realization of a new mass flow sensor using the Peltier effect. The sensor, shaped as a bimetallic printed circuit includes three continuous parallel strips coated with a great deal of metal plated spots. In such a device, the central track performs as a classical thermoelectrical circuitry whose both plated and uncoated parts provide the thermopile junctions. The two outer strips are subjected to electrical currents so as to generate numerous small thermal gradients owing to the Peltier effect. Then, the resulting differences in temperature induce a Seebeck e.m.f. on to the close inner strip acting as a receiver. The thermal coupling between transmitters and receiver tracks depends on any variation of the surrounding environment heat transfert coefficient. Therefore, such a device allows us to detect any shift in physical properties related to the apparent thermal conductivity. In the special case of a steady state fluid, the induced e.m.f. in the receiving track hinges on the thermal conductivity. When the fluid is in relative motion along the sensor, the velocity can be read out as a function of voltage. As an application, the thermoelectric circuit is placed in a tube conducting a fluid flow, in order to design a new mass flowmeter. Experimental results show that when subjected to a steady mass flow rate the e.m.f. remains still, even though the pressure is allowed to vary through the pipe. Actually, the supplied information depends only on the mass flow rate. The main advantage of this measurement method, when compared with classical hot wire devices, is that any change in the surrounding environment average temperature does not induce any significant shift on the output voltage. On the other hand, when operated in a wide temperature range, easy compensating techniques can be used to provide accurate and reliable performance over large temperature variations. 


\section{Introduction.}

L'anémomètre à fil chaud est utilisé depuis longtemps [1] pour les mesures sur les fluides en mouvement. Le principe de mesure consiste à placer un fil chauffé dans un milieu fluide en vue de déterminer les propriétés physiques de ce milieu à partir des variations de la température du fil. Dans la majorité des cas la propriété mesurée est la vitesse du fluide. En fait, puisque la mesure est basée sur le transfert de chaleur entre le fil et son environnement. il est possible de détecter séparément toute variation de température ou de composition du milieu fluide.

Dans un article récent [2], nous avons décrit et caractérisé un nouveau capteur thermoélectrique en forme de fil adapté aux mesures dans les milieux fluides. L'élément sensible est alternativement utilisé pour générer par effet Peltier des écarts de température entre les jonctions thermoélectriques dans un premier temps et pour détecter ces écarts par effet Seebeck dns un deuxième temps. La grande sensibilité d'un tel dispositif aux propriétés du milieu environnant n'est limitée que par la résistance thermique du circuit entre jonctions thermoélectriques.

La possibilité de réaliser des circuits thermoélectriques en forme de circuits imprimés bimétalliques sur support isolant (feuille de kapton), permet d'envisager des structures plus complexes comportant de nombreuses sources entretenant par effet Peltier des gradients de température bidimensionnels sur la surface d'un circuit thermoélectrique en forme de circuit imprimé. La sensibilité de ces dispositifs n'est plus limitée par la résistance entre jonctions thermoélectriques puisque les échanges peuvent être privilégiés suivant la direction transversale du circuit. L'objet de ce travail concerne l'étude expérimentale du couplage entre un circuit thermoélectrique à plusieurs pistes traversé par un courant (circuit émetteur) et un autre circuit thermoélectrique utilisé comme détecteur de gradient thermique placé à proximité, en vue de la réalisation de nouveaux capteurs.

Les résultats présentés montrent que le couplage entre circuits thermoélectriques est très sensible au déplacement relatif du milieu fluide environnant le circuit. A titre d'application un prototype de débimètre massique a été conçu et caractérisé.

\section{Principe de la mesure.}

Les circuits thermoélectriques sont réalisés en forme de circuits bimétalliques maintenus solidaires par un support kapton de $90 \mu \mathrm{m}$ d'épaisseur. Le circuit thermoélectrique de la figure 1 comporte trois pistes parallèles entre elles et effectuant de nombreux aller retour de façon à pouvoir être placé dans une canalisation parallèlement à l'axe $y^{\prime} y$. Chacune des pistes thermoélectriques est constituée par un ruban continu de constantan de $15 \mu \mathrm{m}$ d'épaisseur recouvert de nombreux dépôts de cuivre (ou d'or) de $5 \mu \mathrm{m}$ d'épaisseur. Le circuit a été réalisé par voie électrolytique en protégeant par une résine photosensible les parties du circuit non recouvertes par le dépôt électrolytique de cuivre. Les pistes de largeur $0,7 \mathrm{~mm}$ sont séparées par une distance de $0.4 \mathrm{~mm}$. Sur une longueur de $170 \mathrm{~mm}$ nous avons pu, en repliant le circuit, obtenir 20 ensembles de 6 jonctions thermoélectriques disposés suivant la largeur du conduit. Compte tenu du contraste entre les conductivités électriques du cuivre et du constantan les régions cuivrées se comportent comme des tronçons de cuivre homogène. Les jonctions thermoélectriques sont localisées sur les lignes frontières des dépôts électrolytiques et sont distantes de $2.4 \mathrm{~mm}$. La f.e.m. détectée en circuit ouvert entre les extrémités de chacune des pistes thermoélectriques est représentative de la somme des écarts de température entre jonctions thermoélectriques $[3,4]$. La circulation d'un courant continu dans une piste thermoélectrique provoque à la fois un échauffement par effet Joule et des échanges réversibles par effet Peltier localisés aux endroits où le courant électrique passe du cuivre dans le constantan ou inversement. 


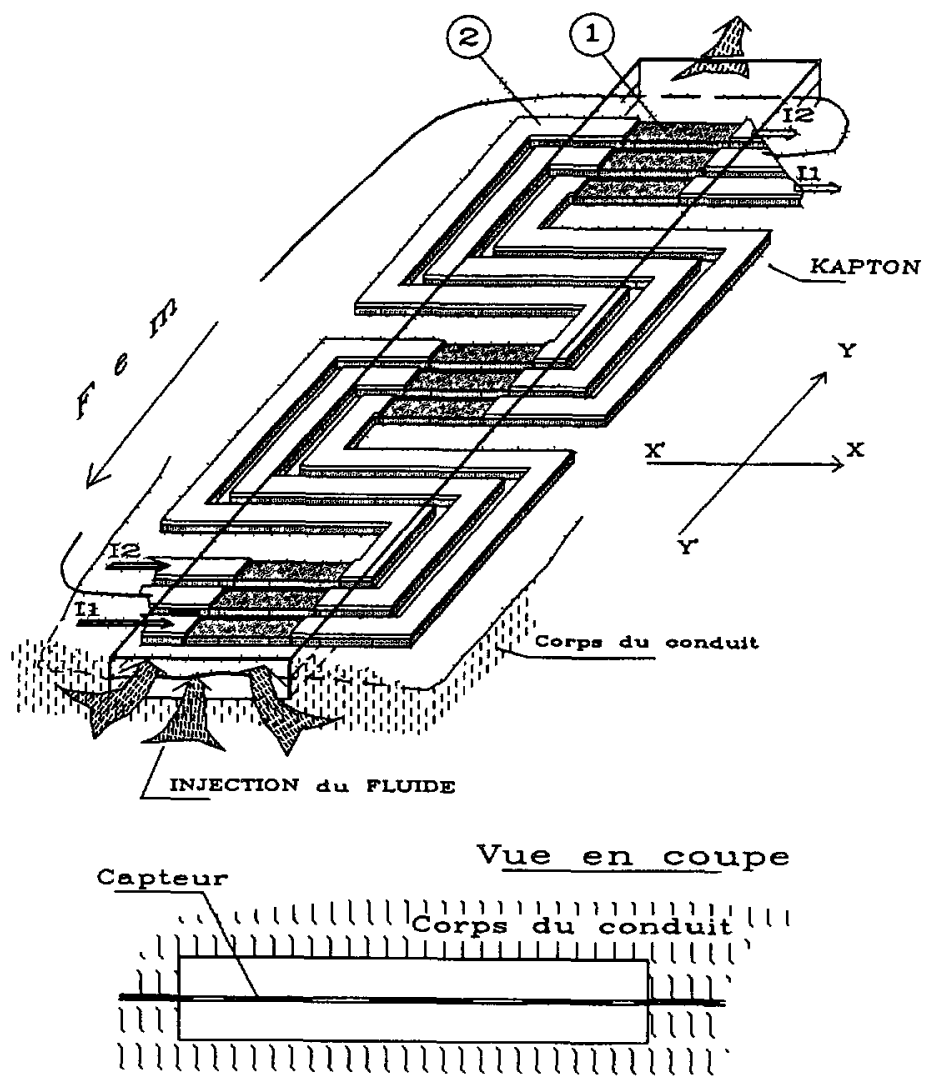

Fig. 1. - Schéma du circuit thermoélectrique placé dans un conduit à section droite rectangulaire.

[Block diagram of the thermoelectric circuit settled in a rectangular straight sectioned duct.]

Sur la figure $2 \mathrm{~b}$ qui représente les lignes de courant électrique dans le circuit, il est facile de voir que les échanges par effet Peltier sont localisés au voisinage des jonctions thermoélectriques où les lignes de courant passent du cuivre dans le constantan et inversement. Pour un courant de $100 \mathrm{~mA}$, la puissance générée par effet Peltier est très faible, de l'ordre de $1,2 \mathrm{~mW}$.

Compte tenu de la faible résistance électrique des pistes, de l'ordre de quelques $\Omega$, l'énergie dissipée par effet Joule est faible $(30 \mathrm{~mW})$ mais relativement importante vis-à-vis de la puissance générée par effet Peltier. Les échanges par effet Joule et par effet Peltier provoquent des variations de température en chaque point du circuit thermoélectrique. Il faut toutefois remarquer que la dissipation par effet Joule, uniformément distribuée entre jonctions thermoélectriques, provoque surtout une élévation de la température moyenne du circuit. Par contre les échanges par effet Peltier, beaucoup moins intenses, mais localisés aux points de jonction entretiennent des écarts de température mesurables entre jonctions thermoélectriques.

Suivant le sens de la circulation du courant dans les pistes latérales, des gradients thermiques sont induits suivant la direction axiale ou perpendiculairement à la direction axiale des circuits. Lorsque le courant circule dans le même sens dans chacune des pistes, les jonctions placées côte-à-côte en suivant le circuit sont des points où il y a alternativement émission et absorption de chaleur. Il en résulte une distribution de gradients thermiques orientés suivant la direction axiale du circuit puisque les transferts suivant la direction transversale sont minimisés (Fig. 3a). 


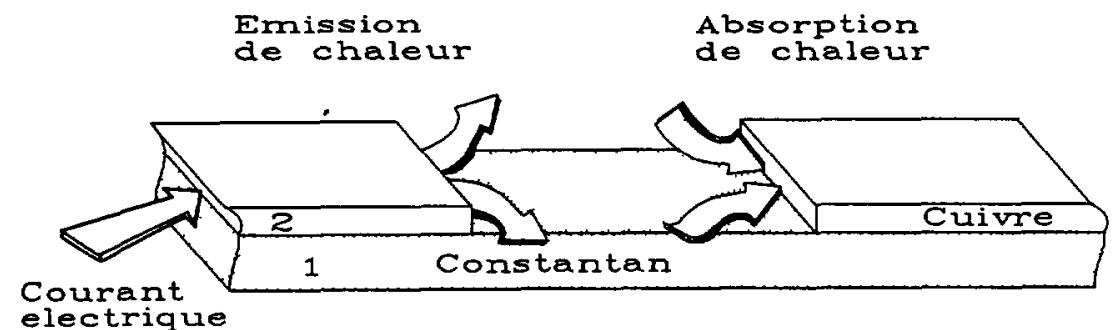

a)

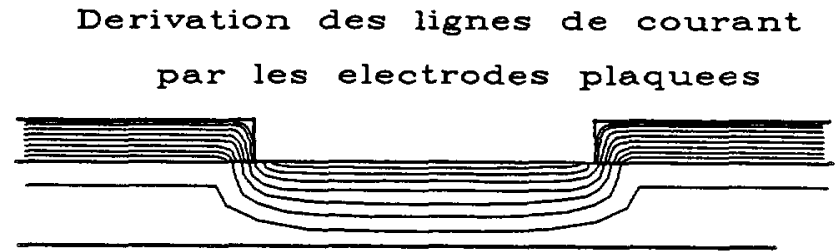

b)

Fig. 2. - a) Génération et absorption de chaleur par effet Peltier au niveau des jonctions thermoélectriques. b) Déviation des lignes de courant électrique par les électrodes plaquées au voisinage des jonctions thermoélectriques.

(a) Heat generation and absorbtion with Peltier effect on thermoelectric junctions. b) Electric current lines deflected by plated electrodes close to the thermoelectricjunctions.]

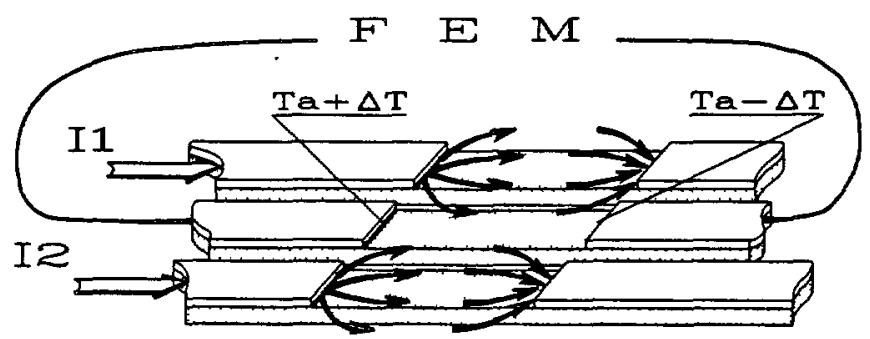

a)

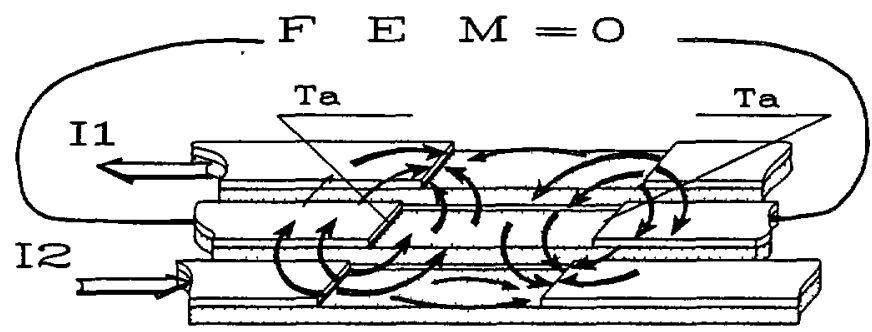

b)

Fig. 3. - a) Gradients thermiques générés par effet Peltier suivant la direction axiale du circuit pour des courants de même sens. b) Gradients thermiques orientés dans la direction transversale.

(a) Thermal gradients induced by Peltier effect along the circuit axis for currents in the same direction.

b) Thermal gradients induced by Peltier effect along the circuit axis for currents in opposite direction.] 
Par contre, lorsque les pistes thermoélectriques latérales sont traversées par des courants de sens contraires aux points de jonctions placés côte-à-côte, les échanges thermiques sont de signes contraires. Les gradients thermiques induits sur la surface du circuit sont pratiquement transversaux (suivant $y^{\prime} y$ ) puisque les transferts suivant la direction axiale du circuit sont minimisés (Fig. 3b). Dans ce cas, les écarts de température sont déterminés par la résistance thermique transversale entre jonctions thermoélectriques. Une piste thermoélectrique a été placée entre les pistes latérales pour mesurer les gradients de température suivant la direction axiale des circuits thermoélectriques. Elle permet de détecter les écarts de température entre jonctions thermoélectriques lorsque les courants circulent dans le même sens dans les pistes latérales. Elle permet de détecter toute dissymétrie du champ de température lorsque les courants circulent en sens contraire puisque les jonctions du circuit détecteur ne sont à la même température que lorsqu'il y a symétrie des échanges thermiques entre deux couples de jonctions consécutives des pistes latérales.

Quel que soit le sens du courant dans les pistes latérales, les différences de température entre jonctions sont influencées par la conductivité du milieu fluide environnant. De plus, la circulation du milieu fluide facilite les transferts d'énergie dans le sens de la vitesse d'écoulement et les rend moins intenses dans le sens contraire. Lorsque les pistes latérales sont traversées par des courants de sens contraires les transferts thermiques sont transversaux. Nous avons représenté figure 4 les lignes de flux entre deux couples de jonctions consécutives. Dans le cas où le fluide environnant est au repos, la seule différence entre les deux figures est le sens des échanges entre jonctions. Compte tenu de la symétrie des échanges, les températures $T_{\mathrm{A}}$ et $T_{\mathrm{B}}$ des jonctions du circuit détecteur sont égales.

Dans le cas où le fluide est en mouvement, le transfert de masse entre jonctions est couplé à un transfert thermique. Les transferts d'énergie représentés figure $4 \mathrm{~b}$ ont été activés dans le

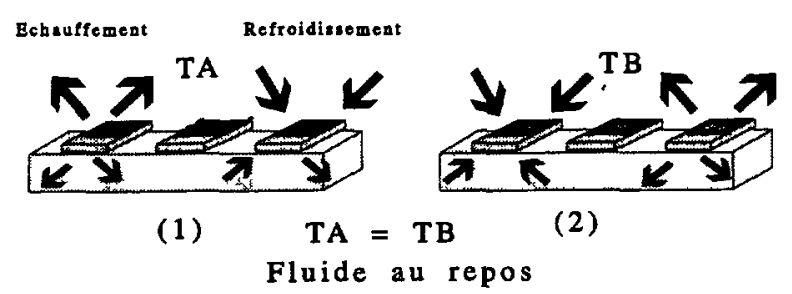

a)
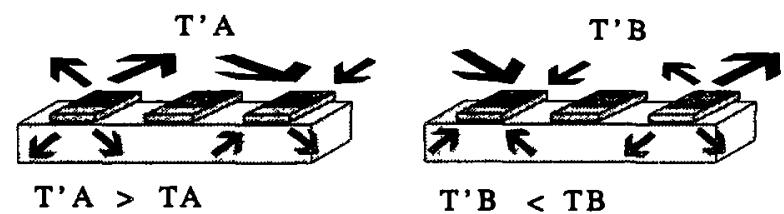

Fluide en mouvement

b)

Fig. 4. - a) Lignes de flux orientés dans la direction transversale dans le plan de section droite de deux jonctions consécutives. b) Influence de la circulation du fluide sur la forme des lignes de flux thermique dans la direction transversale et les températures de jonctions du circuit détecteur.

(a) Flow lines along the transversal axis in the straight sectioned plane of two following junctions. b) Influence of the fluid motion on the thermal flow lines pattern in the transversal axis. Changes of temperatures in the receiving circuit.] 
sens des gradients décroissants et rendus plus difficiles dans le sens des gradients croissants. Il en résulte une modification des températures $T_{\mathrm{A}}^{\prime}$ et $T_{\mathrm{B}}^{\prime}$. L'écoulement du fluide provoque une augmentation de la température $T_{\mathrm{A}}^{\prime}$ et une diminution de la température $T_{\mathrm{B}}^{\prime}$ par rapport aux valeurs obtenues dans un fluide au repos. La différence de température $T_{\mathrm{A}}^{\prime}-T_{\mathrm{B}}^{\prime}$ accessible à la mesure est proportionnelle au débit massique du fluide au voisinage de la surface du circuit qui provoque la modification des températures $T_{\mathrm{A}}^{\prime}$ et $T_{\mathrm{B}}^{\prime}$. En toute rigueur, la f.e.m. détectée est influencée par la température moyenne du circuit puisque :

- la puissance générée par effet Peltier $\left(\alpha_{2}-\alpha_{1}\right) T I$ (où $I$ est l'intensité, $\alpha_{2}-\alpha_{1}$ le pouvoir thermoélectrique, $T$ la température absolue) dépend de la température même si le courant $I$ est maintenu constant, par l'intermédiaire de la température moyenne absolue $T$ et de la dépendance en température du pouvoir thermoélectrique $\alpha_{2}-\alpha_{1}$;

- la sensibilité du circuit détecteur dépend de la température par l'intermédiaire de la dépendance en température du pouvoir thermoélectrique ;

- le couplage thermoélectrique entre les pistes latérales et la piste de détection centrale dépend faiblement de la température moyenne des circuits et n'est pas influencé uniquement par la conductivité thermique apparente du fluide environnant. Pour pouvoir détecter et mesurer toute perturbation, les propriétés physiques du milieu fluide influençant le couplage entre circuits thermoélectriques, il est nécessaire de compenser cette dépendance en température. La méthode classique consisterait à mesurer la température moyenne des circuits et ajuster le courant électrique injecté dans les pistes latérales de façon à annuler les variations de température moyenne des circuits thermoélectriques.

Cette solution est complexe compte tenu des difficultés posées par la mesure de la température moyenne des circuits.

Une autre solution consiste à :

- mesurer comme précédemment la f.e.m. $V_{\mathrm{d}}$ détectée avec des pistes latérales parcourues par des courants de signes contraires. Cette f.e.m. dépend de l'écart de température entre jonctions, de la température moyenne du circuit, de la déformation des lignes de flux entre pistes latérales;

- inverser le courant dans l'une des pistes et mesurer les pistes latérales parcourues par des courants de même sens une f.e.m. $V_{c}$ représentative à la fois des différences de température entre jonctions et de la température moyenne des circuits ;

- les f.e.m. $V_{\mathrm{d}}$ et $V_{\mathrm{c}}$ dépendant toutes deux de la différence de température entre jonctions et de la température moyenne du circuit.

Diviser la f.e.m. $V_{\mathrm{d}}$ par la f.e.m. $V_{\mathrm{e}} / 2$ permet d'obtenir un nombre abstrait indépendant du courant et de la température du circuit (puisque $V_{\mathrm{c}}$ et $V_{\mathrm{d}}$ sont affectées de la même façon par la dérive en température) et que l'inversion du courant ne modifie pas l'échauffement local du circuit par effet Joule.

Nous allons montrer expérimentalement que ce rapport $V_{\mathrm{d}} /\left(V_{\mathrm{c}} / 2\right)$ caractérise la perturbation du champ de température par le milieu fluide et peut être utilisé pour mesurer la vitesse moyenne de déplacement du fluide par rapport au circuit.

\section{Résultats expérimentaux.}

L'objet de la partie expérimentale est de présenter un ensemble de résultats permettant de concevoir et réaliser un nouveau capteur basé sur le couplage entre pistes thermoélectriques. Nous avons réalisé dans ce but un circuit imprimé bimétallique (cuivre/constantan) sur support kapton, dimensionné de façon à pouvoir être placé au milieu d'un conduit (Fig. 1), (de longueur $170 \mathrm{~mm}$, largeur $6,4 \mathrm{~mm}$ et hauteur 2 fois $0,47 \mathrm{~mm}$ ) dont la fonction sera, d'une part de faire circuler un fluide gazeux de propriétés thermophysiques connues parallèlement aux 
surfaces du circuit et d'imposer à la fois des variations de vitesse, de pression $P_{\mathrm{B}}$, de température du fluide dans le conduit.

Pour mesurer et faire varier la pression et le débit volumique dans la canalisation, nous avons utilisé un manomètre et une vanne ajustable (Fig. 5).

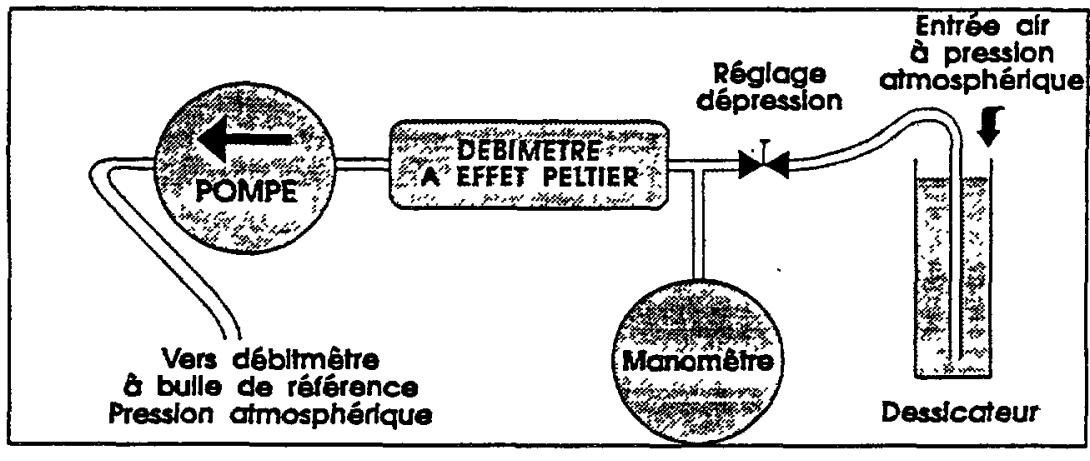

Fig. 5. - Montage utilisé pour faire circuler le fluide à débit massıque constant et faire varier la pression dans la canalisation.

[Apparatus used to obtain a steady mass flow rate when pressure is allowed to vary in the pipe.]

MESURE DES GRADIENTS THERMIQUES INDUITS PAR LE PASSAGE D'UN COURANT. - Lorsque les pistes latérales sont traversées par des courants de même intensité et de même sens, les gradients thermiques générés par effet Peltier sont orientés essentiellement suivant la direction axiale du circuit thermoélectrique. Dans ces conditions, la f.e.m. $V_{\mathrm{c}}$ détectée par la piste détectrice placée entre les pistes émettrices latérales, est représentative des écarts de températures entre jonctions thermoélectriques.

La variation (en valeur absolue) de cette f.e.m. $\left|V_{c}\right|$ en fonction du courant inducteur est représentée figure 6 pour plusieurs types de fluides gazeux. Les courbes obtenues sont linéaires et de plus les valeurs mesurées changent de signe avec le sens du courant inducteur. Les effets d'échauffement du circuit par effet Joule, proportionnels au carré de l'intensité, sont donc négligeables pour des courants inducteurs allant jusqu'à $150 \mathrm{~mA}$.

De plus, le positionnement symétrique des circuits émetteur et détecteur neutralise les effets des gradients Joule existant entre éléments plaqués et non plaqués ce qui permet de limiter l'analyse aux interactions d'origine purement thermoélectrique.

Pour une intensité déterminée, les écarts $\Delta T$ de température entre jonctions thermoélectriques sont d'autant plus importants que la conductivité du fluide est faible. Ce résultat est dû à l'influence de la conductivité du milieu fluide sur la résistance thermique axiale entre jonctions thermoélectriques. Une circulation du fluide à très faible vitesse (débit inférieur à $0,5 \mathrm{~cm}^{3} / \mathrm{s}$ ) a été nécessaire pour obtenir des résultats précis et reproductibles. Les jonctions thermoélectriques étant toutes situées perpendiculairement à la vitesse de déplacement du fluide, le déplacement du fluide dans la canalisation ne provoque pas de variation sensible des gradients thermiques suivant la direction axiale des pistes thermoélectriques. Pour des débits volumiques allant jusqu'à $3 \mathrm{~cm}^{3} / \mathrm{s}$ dans des conditions normales de température et de pression, une variation relative de la f.e.m. inférieure à $3 \%$ a été observée (Fig. 7).

La figure 8 représente l'influence de la température du circuit sur la f.e.m. $V_{c}$ détectée. La variation linéaire avec la température mise en évidence à débit volumique constant est liée à la dépendance en température de la puissance générée par effet Peltier et par la variation en 


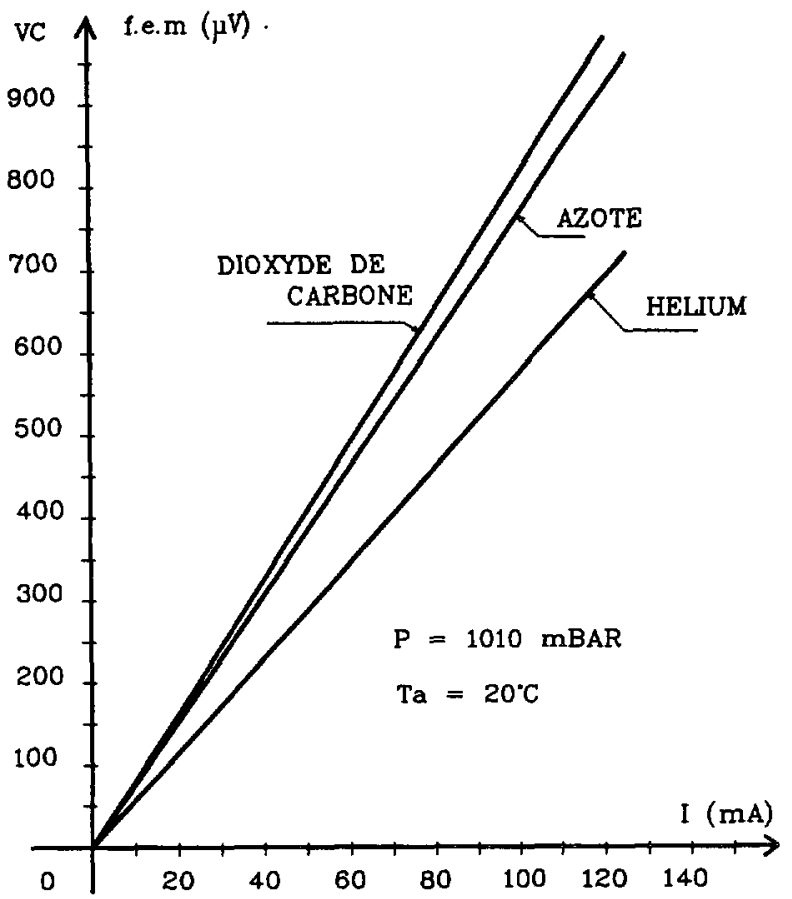

Fig. 6. - Variation en fonction de l'intensité du courant (circulant dans le même sens dans les pistes latérales) des écarts de température entre jonctions thermoélectriques.

[Variation of the differences in temperature in thermoelectric junctions function of both currents flowing along the same axis.]

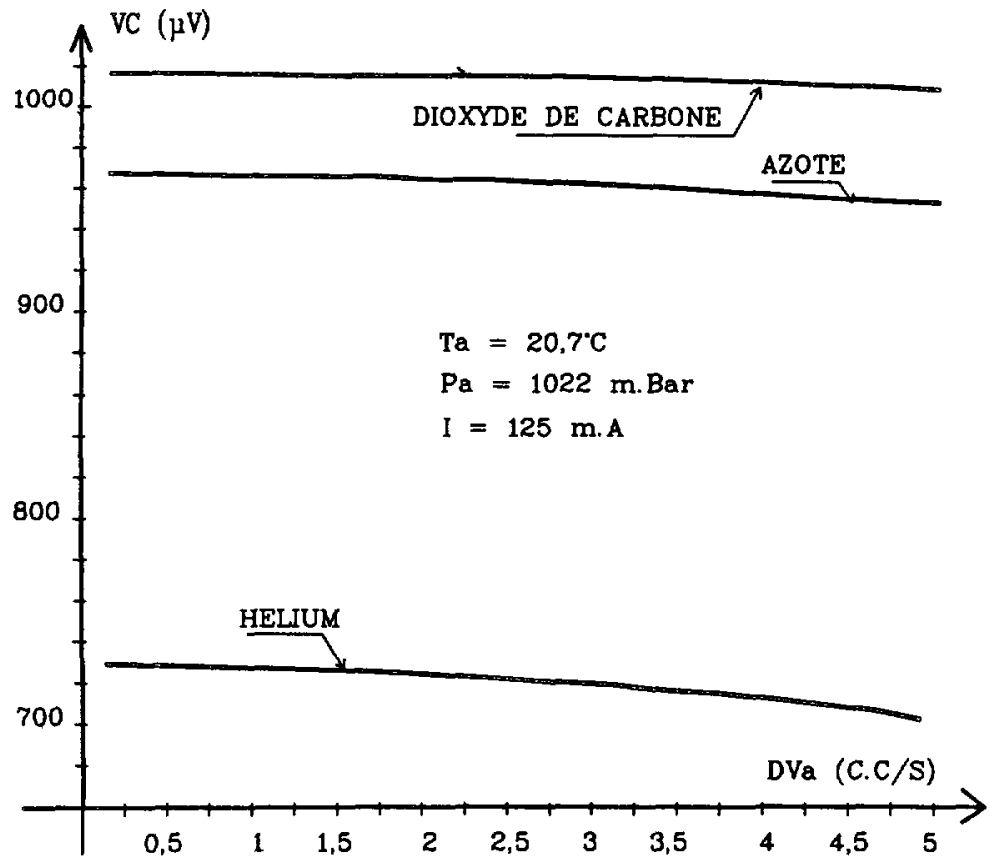

Fig. 7. - Influence du débit volumique sur les écarts de température induits par effet Peltier.

[Mass flow rate influence on the differences in temperature induced by Peltier effect.] 


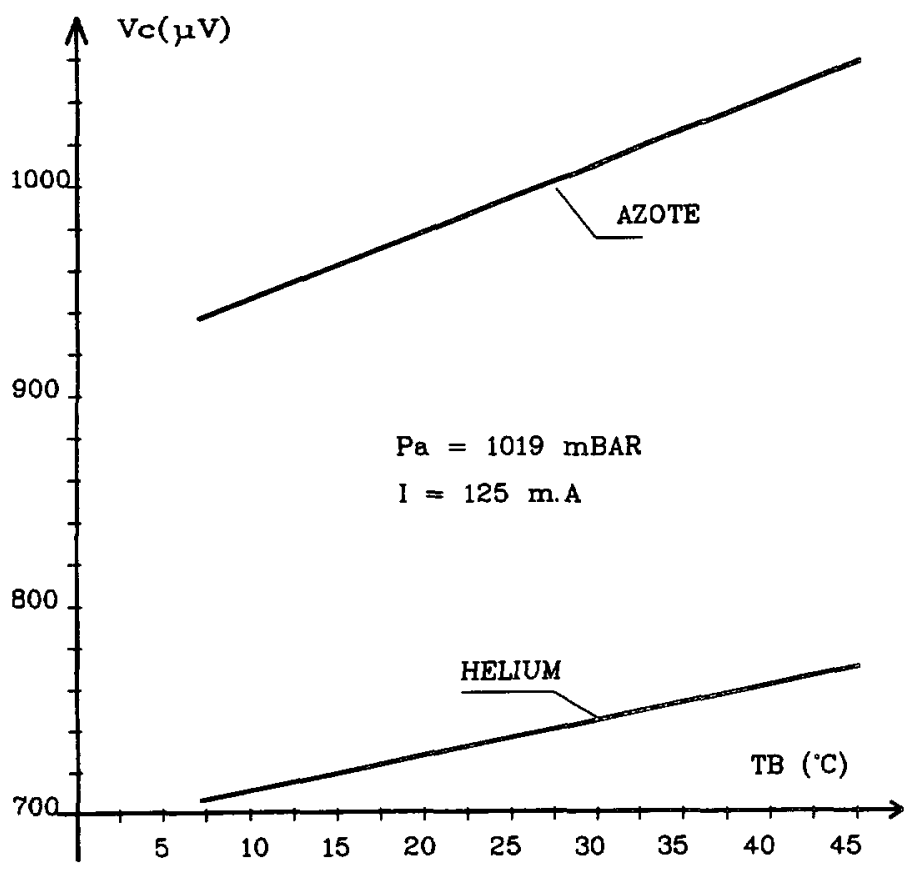

Fig. 8. - Influence de la température sur les écarts de température induits pour un courant de $125 \mathrm{~mA}$ et un débit volumique maintenu constant.

[Influence of the average temperature on the differences in temperature induced by a $125 \mathrm{~mA}$ current under a steady kept volumic flow rate.]

température des caractéristiques physiques du circuit et du fluide environnant. Notons également que la f.e.m. détectée reste constante pour d'importantes variations de pression comprises entre 730 et $1450 \mathrm{mb}$. Ce résultat peut être interprété par la faible influence de la pression sur la conductivité du milieu fluide environnant la gamme de variation considérée.

APPLICATION À LA MESURE DES DÉBITS MASSIQUES. - Les résultats expérimentaux précédents caractérisent l'influence de la température et de la conductivité thermique du fluide sur les gradients thermiques induits par le passage d'un courant électrique dans les pistes latérales.

Nous allons maintenant faire passer des courants de sens contraires dans les pistes latérales de façon à créer des gradients thermiques orientés dans la direction parallèle à la vitesse du fluide et mesurer dans ces conditions la f.e.m. détectée $V_{\mathrm{D}}$ en fonction du débit volumique du fluide dans la canalisation (mesurée dans les conditions normales de pression et température).

A partir des courbes de la figure 9 obtenues sur des fluides de natures différentes, il apparaît clairement que la sensibilité aux variations de débit, c'est-à-dire de vitesse du milieu fluide n'est pas constante. Elle est plus importante dans la région des faibles débits que dans la région des débits importants. De plus, elle change de signe quand le sens de la vitesse d'écoulement s'inverse. Un tel dispositif peut donc être appliqué à la mesure des faibles débits compte tenu de leur sens.

Les résultats présentés montrent clairement que la f.e.m. mesurée dépend du débit volumique dans la canalisation. En fait, puisque le débit volumique est mesuré à température et pression constantes, on pourrait également représenter la f.e.m. détectée en fonction du débit massique dans la canalisation et le problème posé en pratique est de savoir si la f.e.m. 


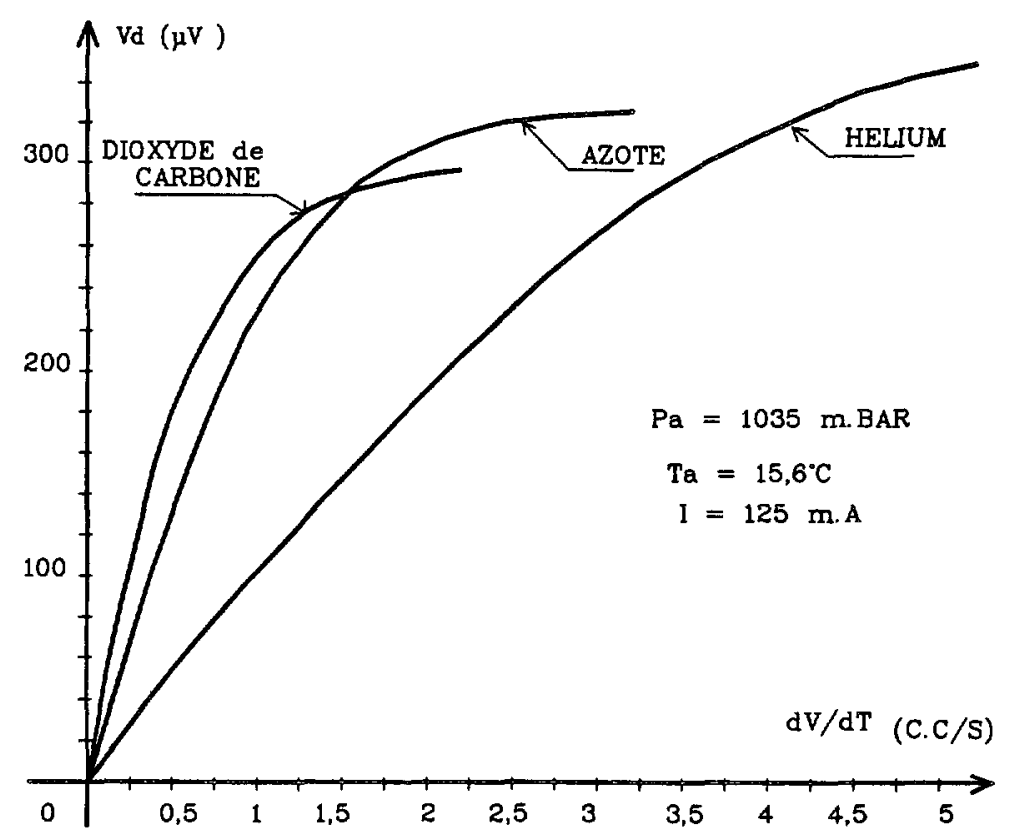

Fig. 9. - Variation de la tension différentielle $V_{\mathrm{d}}$ en fonction du débıt volumique du fluide gazeux dans le conduit.

[Variation of the differential voltage $V_{d}$ function of the fluid gas volumic-flow rate in the duct.]

$V_{\mathrm{d}}$ dépend directement du débit massique ou du débit volumique. Pour répondre à cette question, nous avons maintenu constant le débit massique et fait varier le débit volumique en imposant dans le corps de la canalisation des variations de pression comprises entre 730 et $1450 \mathrm{mb}$. Les résultats expérimentaux ont montré que la f.e.m. mesurée n'est pas influencée par les variations de pression. La f.e.m. $V_{\mathrm{d}}$ est représentative du débit massique dans la canalisation maintenu constant dans les conditions expérimentales. Cette f.e.m. peut donc être utilisée pour mesurer le débit massique du fluide gazeux (Fig. 10).

Suivant ce résultat, la f.e.m. détectée qui ne dépend que du débit massique ne devrait pas être affectée par les variations de température du fluide. En fait, nous avons relevé une faible dérive en température de la f.e.m. détectée à débit massique constant liée à la dépendance en température de la puissance générée par effet Peltier et des propriétés thermiques du capteur (Fig. 11).

COMPENSATION EN TEMPÉRATURE. - L'objectif étant de réaliser un capteur sensible uniquement au débit massique, il est indispensable de compenser la dépendance en température de l'effet Peltier. Compte tenu des résultats de la partie précédente, la méthode la plus simple consiste à calculer le rapport sans dimension :

$$
N=\frac{V_{\mathrm{d}}}{\left(V_{\mathrm{c}} / 2\right)}
$$

qui ne dépend que du débit massique dans la canalisation et a l'avantage de n'être influencé ni par les variations du courant électrique, ni par les variations de température moyenne des circuits. 


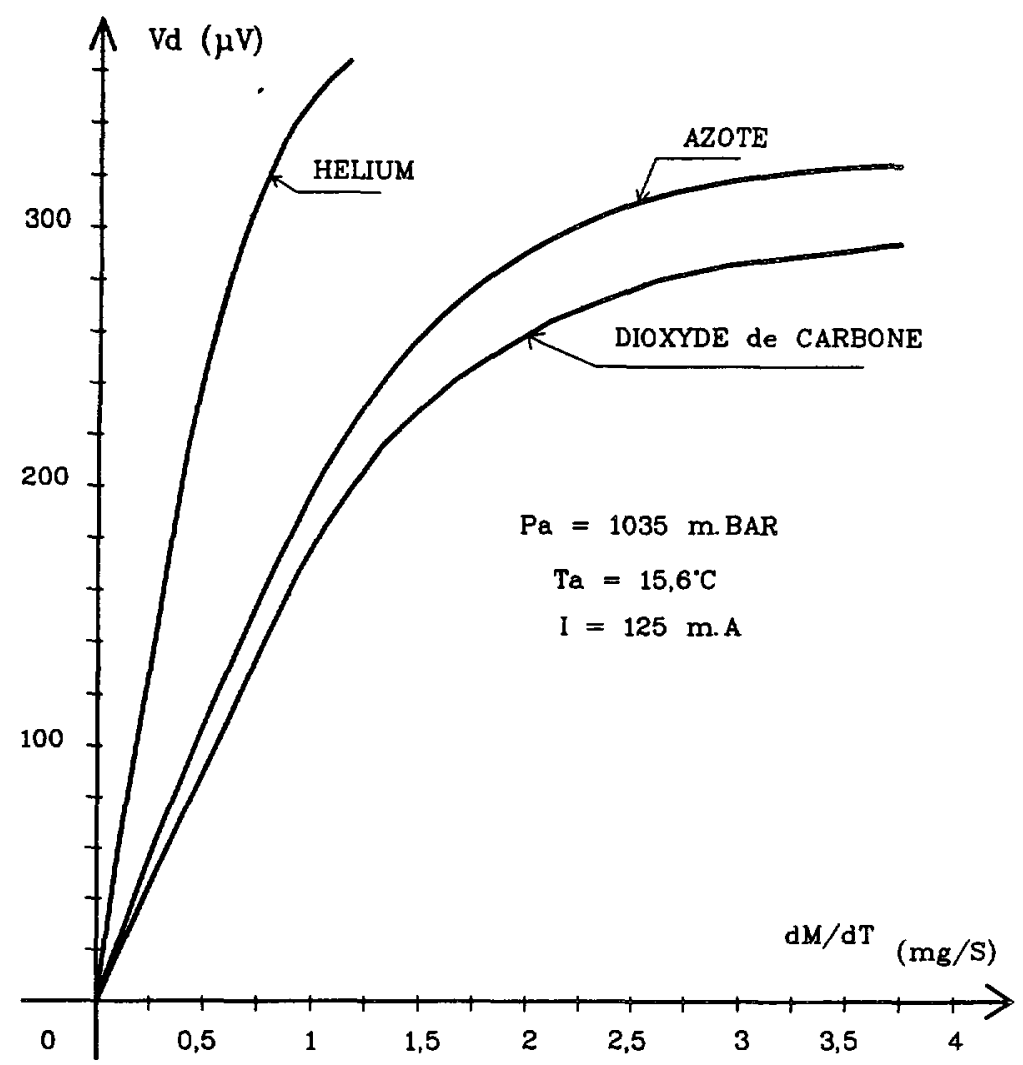

Fig. 10. - Représentation de la f.e.m. mesurée en fonction du débit massique.

[Experimental e.m.f. as a function of mass flow rate.]

Toutes les mesures effectuées entre $6{ }^{\circ} \mathrm{C}$ et $45^{\circ} \mathrm{C}$ présentées figure 12 ont pu être placées sur une même courbe d'étalonnage.

Cette courbe d'étalonnage permet de mesurer le débit massique dans la canalisation quelles que soient les conditions de température et de pression puisque la courbe d'étalonnage reste inchangée pour des variations relatives de ces grandeurs aussi importantes que $\Delta P / P=50 \%$, $\Delta T / T=10 \%$.

CONSTANTE DE TEMPS. - Une autre caractéristique importante d'un capteur de débit massique est son temps de réponse à une variation brusque en échelon du débit dans la canalisation. Pour caractériser le comportement en régime transitoire, nous avons relevé les réponses indicielles des f.e.m. $V_{d}$ et $V_{c}$ lorsqu'une variation brusque de débit est imposée dans la canalisation. Les variations brusques de débit ont été réalisées en pinçant la canalisation souple utilisée pour extraire le fluide. Les relevés normalisés représentés figure 13 caractérisent le capteur en régime transitoire. Les courbes obtenues pour deux fluides montrent que le temps de réponse est d'autant plus faible que la conductivité du fluide est élevée $(50 \mathrm{~ms}$ pour l'hélium, $100 \mathrm{~ms}$ pour l'azote). Comme en régime permanent, la f.e.m. ne dépend pas du sens d'écoulement du fluide alors que $V_{\mathrm{d}}$ change de signe avec le sens de la vitesse d'écoulement. 

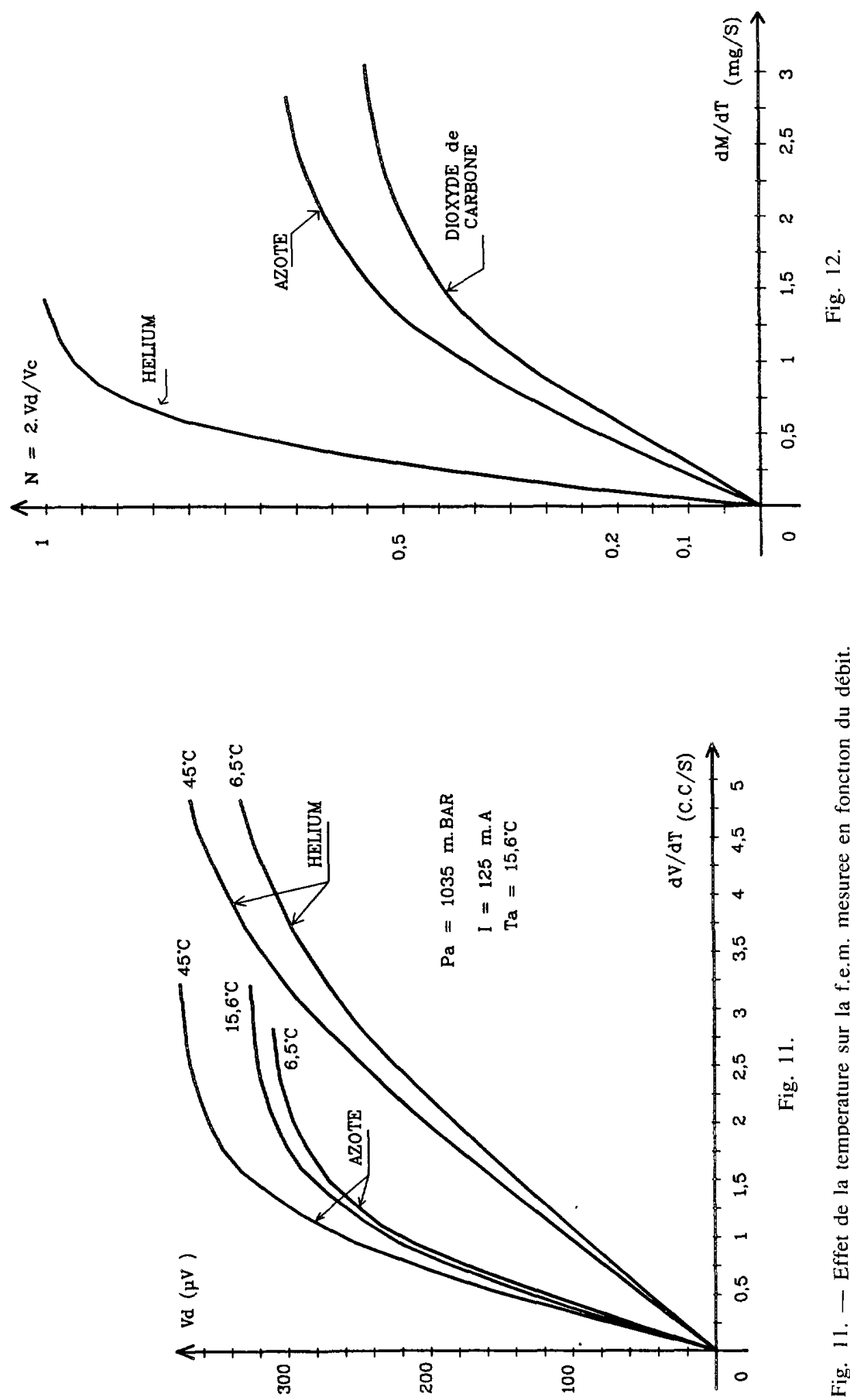

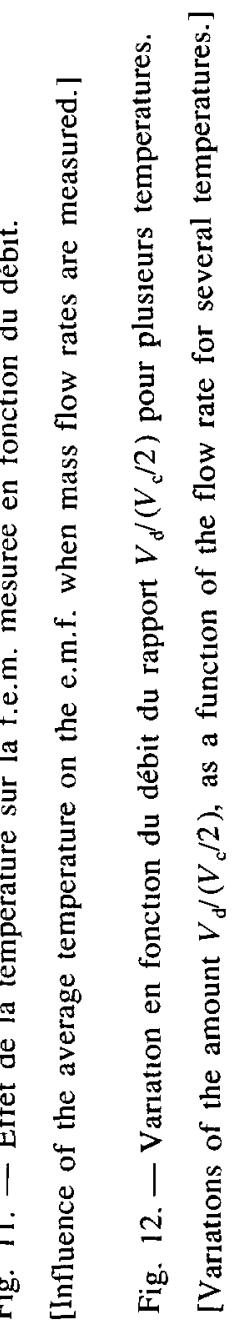




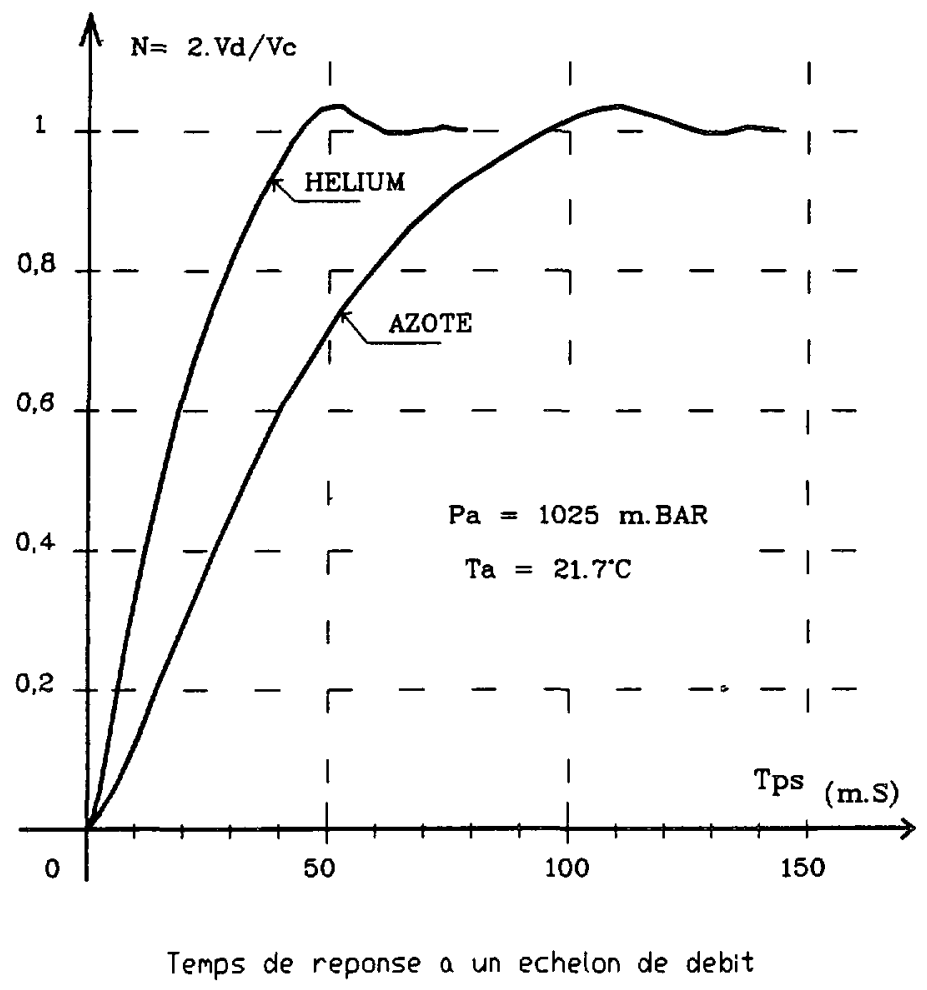

Fig. 13. - Réponse du capteur à une variation en échelon du débit.

[Sensor response to a step-change of mass-flow rate.]

\section{Conclusion.}

L'étude expérimentale des gradients thermiques entretenus par effet Peltier sur la surface d'un circuit imprimé bimétallique nous a permis de concevoir et réaliser un nouveau débimètre massique. Ce capteur est sensible particulièrement dans la région des faibles débits. Un développement du travail présenté pourrait être l'application de cette méthode de mesure aux débits de liquide en augmentant l'intensité du courant dans les pistes thermoélectriques latérales.

\section{Bibliographie}

[1] Bruun M. M., Kahn M. A., Hl Kaijien M. M.. Farad A. A., Velocity calibration for hot wire anemometry, J. Phys. E. Sci. Instr. 21 (1988) 225-32.

[2] Leclercq D., Thery P., Utilization of the Peltier effect for accurate measurement of a fluid property. Application for designing new sensors, Measurement Science and Technology, 4 (1993) 158163.

[3] Herin Ph., Thery P., Measurement on the thermoelectric properties of thin layers of two metals in electrical contact. Application for designing new heat flow sensors, Measurement Science and Technology, 3 (1992) 495-500.

[4] Hladik J., Métrologie des propriétés thermophysiques des matériaux (Edition Masson, 1990). 